\title{
Mujeres, Dones, Mulleres, Emakumeak. Estudios sobre la historia de las mujeres y del género
}

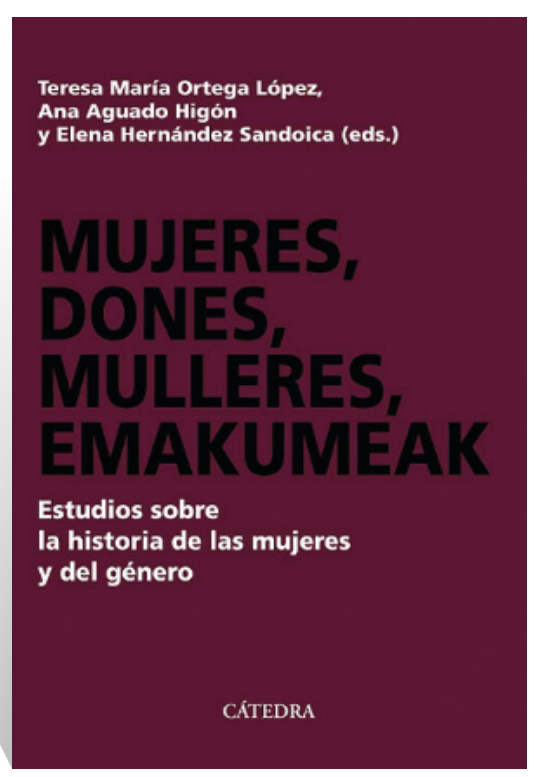

FICHA BIBLIOGRÁFICA

Teresa Ortega López, Ana Aguado Higón, Elena Hernández SAndoica, Mujeres, Dones, Mulleres, Emakumeak: Estudios sobre la historia de las mujeres y del género, Madrid, Cátedra, 2019, 421 págs, ISBN 978-84-376-3977-2

\section{Encarnación Lemus I Universidad de Huelva}

ESTA OBRA EXTENSA INCLUYE VEINTE CAPÍtulos en los que sus autoras reflexionan sobre la historia del feminismo y los estudios de género en España y en otros espacios -en concreto Cuba en la última etapa de su historia colonial y la Rusia de los Soviets-. Como ya explican sus editoras, la obra muestra en su conjunto la diversidad geográfica, generacional y de temática que incluyen estas disciplinas, pero, sobre todo, en mi opinión, lo que mejor se refleja es la riqueza teórica y la pluralidad de enfoques metodológicos y de líneas de investigación, de ahí que la Revista de Historiografía sea el lugar apropiado para este comentario.

Esos veinte capítulos quedan organizados en cuatro bloques: Las mujeres y el proceso de modernización; Inclusión y exclusión; Feminismo, antifascismo y transgresión; Relacio- 
nes de género y mujeres: trayecto y desafíos de un nuevo sujeto historiográfico. Sin embargo, como el origen de este trabajo radica en un merecido reconocimiento a la historiadora Mary Nash y a su obra, la diversidad entre las aportaciones resulta, en realidad, más bien aparente, puesto que el común argumento y, por tanto, la base temática consisten precisamente en el análisis de la renovación historiográfica realizada por esta investigadora y sus aportaciones durante más de cuatro décadas, desde su tesis -La mujer en las organizaciones políticas de izquierda en España, 1931-1939- defendida en 1977. En definitiva, los grandes ejes de la obra de Mary Nash funcionan también como principales líneas de contenido en estos estudios, proporcionando coherencia a pesar de la dispersión de los capítulos.

Así, se observa una línea argumental en torno al crecimiento conceptual de las mismas disciplinas de feminismo y género y se destaca su labor pionera para ir extendiendo en la década de los setenta la obra de las primeras pensadoras, filósofas, feministas clásicas que han ido construyendo las teorías y conceptos en la historia de las mujeres, a partir de Simone de Beauboir, Juliet Mitchell, Zillah Einsenstein, Kate Millet o Julia Kristeva, entre otras muchas. Y, tras ellas, otras más, como Joan Scott y Gisela Bock, cuyas aportaciones demostraron el valor de categorías como sexo, género y feminismo para el análisis histórico y, así, otras sucesivas renovaciones: la incorporación posterior de los estudios de Karen Offen y Edward P. Thompson, que entendieron el feminismo como movimiento social abierto, no excluyente, no uniforme y que colocan como objetivo de la disciplina no sólo la visibilización de las mujeres en la historia, sino la comprensión de los mecanismos de reproducción de desigualdad, de las relaciones de poder entre mujeres y hombres. Y, al fin, derribar ya en el siglo XXI otra frontera más, al profundizar no en el feminismo sino en "los feminismos en sus contextos"; historiar no desde un feminismo occidentalista, sino desde la experiencia postcolonial, con feminismos que colocan sus centros en África o Asia para focalizar conjuntamente desigualdades de géneros, culturas, razas u oportunidades. Estos y otros avances que Miren Llona y Nerea Aresti han ido extrayendo de las distintas obras de Nash hasta llegar a "El desafío de la diferencia" y "Los feminismos históricos: revisiones y debates"

En la misma línea de herencia teórica y metodológica se sitúa el estudio de Mercedes Arbaiza, Dones en Transició: el feminismo como acontecimiento emocional, en el que, con su análisis sobre emociones y sentimientos, los "malestares" insertos en un grupo de entrevistas, depositadas en AHOA Archivo de la Memoria, retoma otro de los grandes trabajos de Nash, Dones en Transició2. Y, por supuesto, la presentación que realiza Cándida Martínez de la revista Arenal, recordando, con el afecto los 25 años de dirección conjunta que la revista, como una extensión de las renovaciones que estoy comentando, ha acogido e impulsado los grandes campos de la disciplina: la aplicación y extensión de un nuevo bagaje conceptual y

1. M. Nash y D. Marre (eds.) El desafío de la diferencia: representaciones culturales e identidades de género, raza y clase, Bilbao, Universidad del País Vasco, 2003 y M. Nash, “Los feminismos históricos: revisiones y debates”, en CENARRO, Á. e ILLION, R. (eds.), Feminismos: contribuciones desde la historia, Zaragoza, Prensas Universitarias de Zaragoza, 2014, pp. 27-50

2. M. Nash, Dones en Transició. De la rèsistencia política a la legitimitat feminista: les dones en la Barcelona de la Transició, Barcelona, 2007.

Revista de historiografía 33, 2020, pp. 282-286 
metodológico; la apropiación del espacio público: estrategias, acciones y nociones, formas, prestando, por ejemplo, mucha atención a la intervención de las mujeres en los asuntos públicos -con monográficos como Mujeres, paz y regulación de los conflictos- o a las transgresiones y espacios de libertad, con temáticas como Mujeres viajeras, aventureras, estudiosas y turistas o Cuerpos, discursos e identidades.

Existe otro poderoso eje de reflexión, que cobra si cabe mayor envergadura filosófica en este marco de feminismos abiertos hacia el análisis de las desigualdades, las frágiles confluencias entre Socialismo radical y Feminismo y, más aún, entre Marxismo y Feminismo. En el fondo, cómo combinar los motores de la lucha de clase y el feminismo, una indagación que arranca de la poderosa vinculación establecida por Mary Nash entre el feminismo y la lucha emancipadora y revolucionaria de las mujeres de izquierda en su clásica Rojas ${ }^{3}$. Con ese marco, Ana Aguado analiza el feminismo de Ana Cambrils (1877/1939) dentro del socialismo, una fusión que la misma Cambrils condensó en el título de su obra Feminismo socialista (Valencia, 1925 con prólogo de Clara Campoamor). Cambrils defendió siempre un feminismo de clase frente a los movimientos religiosos que surgían como alternativa, y Aguado señala que ese feminismo socialista fue compartido por otras mujeres socialistas como Virgini González, Margarita Nelken, María Lejárraga o Julia Vega. En el marco cronológico del primer tercio del siglo en España, estas feministas confiaban en la alianza entre socialismo, libertad y democracia, en un todo conjugable con el feminismo, como instrumento de liberación y reivindicación de ciudadanía e igualdad política para las mujeres, pero básicamente para la emancipación de las mujeres trabajadoras.

Por otra parte, también la combinación entre marxismo revolucionario y feminismo reaparece en distintos capítulos, pero es central, a pesar de los diversos espacios y cronologías, en el estudio de Elena Hernández Sandoica al enfocar las evoluciones de las revolucionarias soviéticas. Una disyuntiva análoga es retomada por Mónica Moreno para la España de los setenta en el marco de la militancia de ultraizquierda con la pregunta ¿Cómo se ajustaron las mujeres al sujeto revolucionario? Se compone, así, un eje que vertebra la historia de la lucha política de las mujeres, rota entre una teoría que sentaba la idea de que la cuestión de la mujer se disuelve en la cuestión social y una práctica que, como asevera Hernández Sandoica, se reveló incapaz de "liberarlas a ellas mismas y a sus congéneres, como mujeres, de la sumisión patriarcal".

Es la misma disrupción que Mercedes Yusta sitúa en el centro, al enfocar la ruptura entre el Antifascismo y el Feminismo una vez terminada la II Guerra, dejando manifiesta la imposibilidad de "pensar conjuntamente las desigualdades de género y clase. Para la línea oficial de la Federación Democrática Internacional de Mujeres a partir de 1946, el feminismo es reducido a su condición de ideología burguesa".

En contraste con ello, ronda la idea de que, en el breve marco de la coyuntura de la España republicana y en guerra, fue viable la asociación entre la lucha revolucionaria de las anarquistas y la emancipación de la mujer, dentro de la organización libertaria Mujeres Libres, fusión que planteó Nash como "anarcofeminismo" y retoma Dolores Ramos a través de la cita de la obra de

3. M. Nash, Rojas: las mujeres republicanas en la Guerra Civil, Madrid, 1999 y 2016. 
Colectivo Arcàdia, Eulalia Vega et al. ${ }^{4}$, pero la experiencia queda suscrita al período de cambio y no alcanzó el tiempo de la "normalidad histórica", si es que existe algo así.

Tras esa conceptualización densa, el tercer eje lo compone ese reconocido enunciado de que lo personal es político que encierra debajo de su sencillez la mayor hondura al replantear la historia de las mujeres fundiendo los planos y, de camino, marca la tarea de llenar de mujeres los espacios históricos o de restituir el papel de las mujeres como agentes históricos. En este objetivo, resulta más que deliciosa la personalísima reconstrucción que realiza Pilar Ballarín a través de los libros de su propio aprendizaje: Flora o la educación de la niña, Guirnaldas de la Historia, Cuando las grandes mujeres eran niñas y las Enciclopedias. En esos textos, además de la reflexión sobre los valores y la ideología que transmitían, dentro del amplio campo de cultura y feminismo, se nos señala por dónde empezar a recuperar la historia de las mujeres. Y a la tarea de recuperar en el tiempo, Susanna Tavera añade la de hacerlo en el espacio y sitúa a las primeras feministas en los barrios de la Barcelona finisecular y de las primeras décadas del XX.

Esencial entre lo personal y lo político ha sido para las mujeres la cuestión del divorcio, a ello presta atención Rosario Ruiz Franco, pulsando el clima social ante el divorcio desde ese primitivo Club de Matrimonios Mal Avenidos nacido en 1903 -cuyo nombre nos hace sonreír ante lo "políticamente correcto" de la denominación-, que llevó a Carmen de Burgos hasta la final publicación de su El divorcio en España, que cito por la circunstancia de que fue publicado en la imprenta de Viuda de Martínez Serra en 1904, y, tras ese sondeo pionero, el de enero de 1932, en el que Ruiz Franco destaca el "tono irónico y superficial” de muchas entrevistas que representan cómo se entendía el tema en una parte importante de la sociedad. También prestó mucha atención a la Ley de divorcio la Unión Republicana Femenina, reforzando la posición del laicismo frente a la crítica de la confesionalidad católica ante las elecciones de 1933, como analiza Luz Sanfeliu. Por otra parte, el comportamiento de las mujeres granadinas en esas elecciones de 1933 resulta detallado minuciosamente en la aportación de Rosa Ma Capel: votó un 69,20\% del censo y, tras un pormenorizado análisis cuantitativo, concluye que el apoyo de los granadinos y las granadinas a las distintas candidaturas no dependió tanto "del sexo de quienes votaban como de razones culturales, económicas y sociales en las que hay que profundizar".

Partiendo también de la idea de que la redefinición de lo público y lo privado es clave en la trayectoria de emancipación de las mujeres, Ángela Cenarro termina dirigiendo una mirada hacia la Sección Femenina de Falange y a su recuperación "obsesiva" del discurso de separación de roles y espacios atribuidos a los sexos y de la jerarquía entre ellos y cómo se acude, no obstante, al recurso de la excepcionalidad para "legitimar el lugar privilegiado de las falangistas en el Nuevo Estado franquista y representar sin riesgos su presencia en el espacio público". En el caso contrario, a las que fueron represaliadas por haberlo ocupado para disentir, además, de lo tradicional se dirige la atención de Montserrat Duch: a las maestras catalanas represaliadas por las Comisiones Depuradoras del Magisterio.

4. Colectivo Arcàdia, E. Vega et al., Mujeres Libres y feminismo en tiempos de cambio, Madrid, Fundación Anselmo Lorenzo, 2016.

Revista de historiografía 33, 2020, pp. 282-286 
Si en algún ámbito cuenta menos la dimensión pública del espacio femenino es en el mundo agrario, poco privilegiado en los estudios históricos en general y en los de género también. Como dice Teresa Ortega, funciona junto al "androcentrismo" un "urbanocentrismo"; sobre esa base, su investigación asume que las mujeres rurales son un "colectivo estratégico y potenciales agentes de cambio social y político”. El interés de este tipo de indagación radica, además, en su posible proyección en el mundo de hoy, para comprender y actuar sobre la potencial transmisión político-social de los estudios feministas sobre los roles que cumplen y pueden desempeñar mujeres rurales contra desigualdades económicas y patriarcales en países del Tercer Mundo.

Una reflexión final me lleva a retomar el principio, la vastedad de la obra de Mary Nash y la vivacidad y el intenso seguimiento que su tarea pionera ha ido recogiendo por historiadoras de generaciones distintas que enriquecen y multiplican los caminos para que todo tiempo histórico sea tiempo de mujeres y todos los lenguajes sirvan para desentrañar el componente de género, como en los estudios culturales de las genealogías femeninas en la arquitectura griega de $\mathrm{M}^{\mathrm{a}}$ Dolores Mirón o en la exégesis del Amor cortés en el primer tercio del siglo XX de José Javier Díaz. En suma, bajo el paraguas de Rojas sitúa Vicenta Verdugo Martí su análisis de dos militantes valencianas: Rosa Estruch Espinós, comunista y alcaldesa con 24 años de Vilallonga, y de Pilar Soler, de la Agrupación de Mujeres Antifascistas, AMA. Al morir la primera, como recoge Estruch, Pilar escribió: "Hay vidas que ocupan tales espacios que a la hora de recordar y para contar su vida, resulta casi imposible poderlo hacer con todo lo deseado...". Exactamente eso, que hay muchas vidas que contar: cómo hacerlo y para qué, eso es lo que Mary Nash nos enseña y lo que este libro demuestra. 